\title{
Pulmonary Cryptococcosis Presenting as Endobronchial Lesions in a Patient under Corticosteroid Treatment
}

\author{
Yuichi Inoue ${ }^{1}$, Yoshitsugu Miyazaki ${ }^{2}$, Kohichi Izumikawa ${ }^{2}$, Katsunori Yanagihara ${ }^{2}$, \\ Hiroshi Kakeya ${ }^{2}$, Toyomitsu Sawai ${ }^{1}$, Yoichi Hirakata ${ }^{2}$ and Shigeru Kohno ${ }^{2}$
}

\begin{abstract}
We report a case of pulmonary cryptococcosis in an immunocompromised patient who had focal endobronchial lesions. When we first examined her, she complained of a productive cough that had lasted for two months. Seventeen months prior to this examination, she had been diagnosed with Sjogren syndrome and Sweet syndrome. Since that diagnosis, her condition had been maintained with low-dose prednisolone. We performed a chest CT scan, which revealed a mass, $3 \mathrm{~cm}$ in diameter, in the upper lobe of her left lung. A bronchoscopic examination revealed 3 white, elevated lesions in the upper lobe bronchus of her left lung. After 40 days of treatment with fluconazole, the shadow of her lung mass decreased in size to that of a scarred lesion and her white, elevated bronchial lesions disappeared.
\end{abstract}

Key words: endobronchial cryptococcosis, pulmonary cryptococcosis, immunocompromised patient, bronchoscopy, lung mass, corticosteroid

(DOI: 10.2169/internalmedicine.46.6249)

\section{Introduction}

There are only a few rare descriptions (1-3) of cryptococcus presenting itself as endobronchial lesions, which cause volume loss in the lung or lung collapse. We report a case of pulmonary cryptococcosis in an immunocompromised patient who had focal endobronchial lesions.

\section{Case Report}

A 54-year-old woman was referred to us for further evaluation of an abnormal shadow that was present on an Xray of her chest.

At the time of her first consultation, she was hospitalized in a dermatology ward suffering from skin disease (pustular vasculitis). When she first entered the dermatology ward, she was given prednisolone at an initial dose of $40 \mathrm{mg}$. It was reduced by $20 \mathrm{mg} /$ day 4 weeks after hospitalization, and to 7 weeks after hospitalization. Her condition was being maintained with a $7.5 \mathrm{mg}$ daily dose of prednisolone. When we first examined her 10 weeks after hospitalization, she complained of a productive cough that had lasted for 2 months. Seventeen months prior to this examination, she had been diagnosed with Sjogren syndrome and Sweet syndrome. Since that diagnosis, her condition had been maintained with low-dose (5.0-7.5) prednisolone. The patient presented no abnormal physical findings, except for skin eruptions.

In a routine blood test, we obtained the following abnormal results: her white cell count was $10200 \mathrm{~mm}^{3}$; her Creactive protein level (CRP) was $5.77 \mathrm{mg} / \mathrm{ml}$; and her erythrocyte sedimentation rate was $89 \mathrm{~mm} / \mathrm{hr}$ during the first hour (Table 1). Her immunological test results were close to normal, except for a negative PPD skin test (Table 2).

A chest X-ray revealed a mass-like shadow in the upper lobe of her left lung and an elevation of her diaphragm due to a compression fracture of the thoracic and lumbar spine resulting from long-term administration of corticosteroids (Fig. 1). A chest CT scan revealed a mass, $3 \mathrm{~cm}$ in diameter, in the upper lobe of her left lung, and left hilar and mediastinal lymphadenopathy with several small nodules scattered throughout both of her lungs (Fig. 2).

A bronchoscopic examination revealed 3 white, elevated

\footnotetext{
${ }^{1}$ Department of Internal Medicine, Isahaya Insurance General Hospital, Nagasaki and ${ }^{2}$ Second Department of Internal Medicine, Nagasaki University School of Medicine, Nagasaki

Received for publication September 20, 2006; Accepted for publication December 15, 2006

Correspondence to Dr. Yuichi Inoue, naika02@ hospital-isahayasougou.jp
} 
Table 1. Laboratory Data

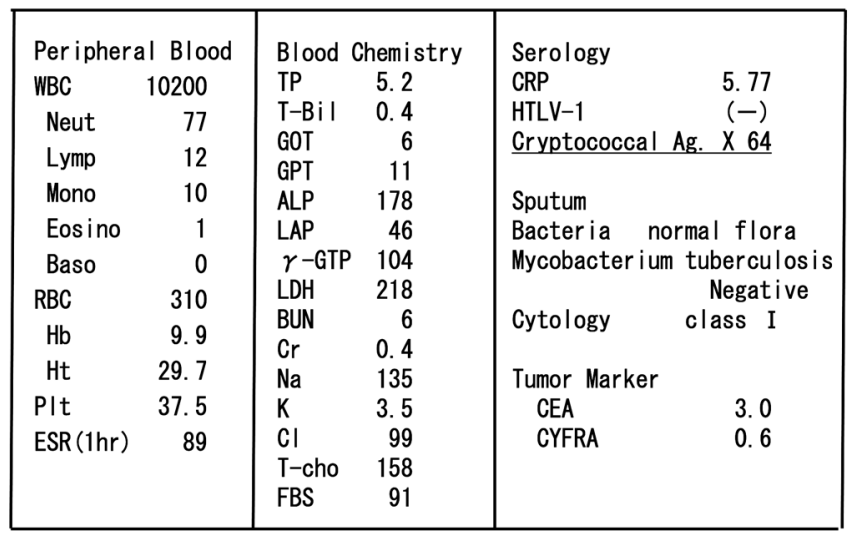

Table 2. Immunological Tests



lesions in the upper lobe bronchus of her left lung (at the spur of the upper lobe bronchus, at the orifice of the lingular and at the orifice of the left bronchus $\left(\mathrm{B}^{1+2}\right)$, all of which were subsequently biopsied (Fig. 3).

A histopathological examination of the transbronchial biopsy specimens revealed the presence of granulomatous lesions, including cryptococcal organisms, marked neutrophil infiltration, and some epithelioid cells and Langhans giant cells (Fig. 4).

The serum was positive for cryptococcal antigen, with a titer of $1: 64$. Based on these results, we diagnosed her as having endobronchial cryptococcosis. After forty days of treatment with fluconazole, at a daily dose of $400 \mathrm{mg}$, the shadow of her lung mass decreased in size to that of a scarred lesion and her white, elevated bronchial lesions disappeared (Fig. 5). Inflammation (white blood cell count, erythrocyte sedimentation rate, and CRP level) was almost normalized 5 weeks after treatment, and serum cryptococcus antigen decreased, with a titer of $1: 2$, within 5 months. Fluconazole treatment was stopped 5 months after it was started.

\section{Discussion}

Cryptococcosis is caused by the yeast-like fungus, Cryptococcus neoformans, which has a world-wide distribution. The USA and Australia have been described as having the highest prevalence of Cryptococcus neoformans (4), and more than one hundred cases of cryptococcosis have also

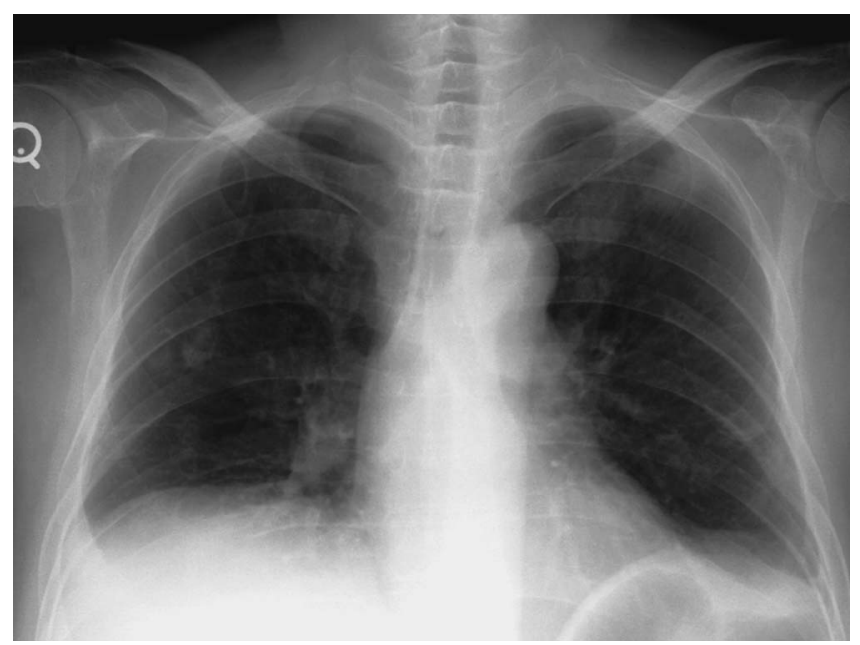

Figure 1. A chest X-ray showing a mass-like shadow in the upper lobe of the left lung and several small nodules scattered throughout both lungs.

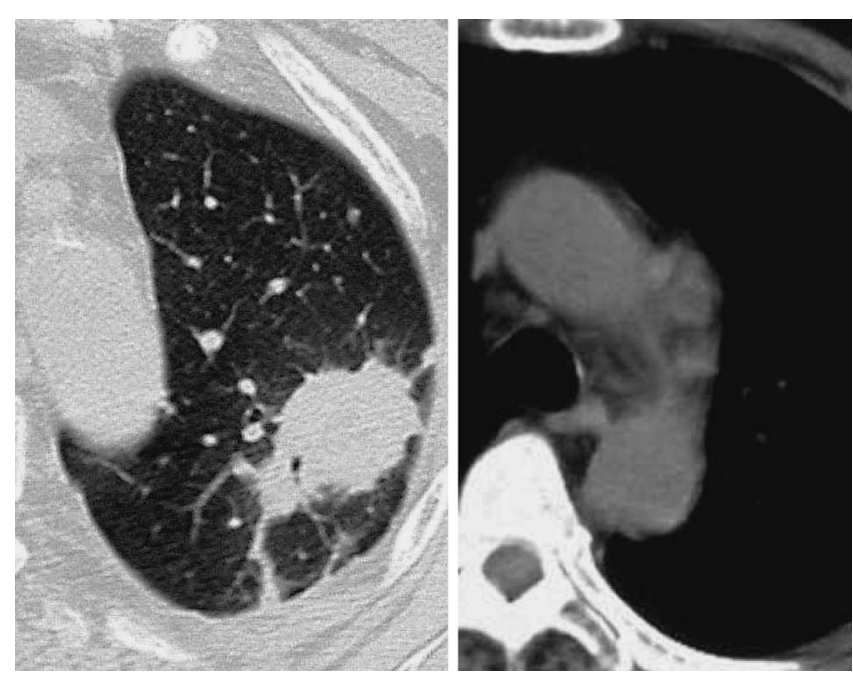

Figure 2. A chest CT scan revealing a mass, $3 \mathrm{~cm}$ in diameter, in the left upper lobe and left hilar and mediastinal lymphadenopathy.

been reported in Japan. Although pulmonary cryptococcosis occurs both in healthy individuals and in immunocompromised patients, it is frequently recognized as an opportunistic pathogen (50-80\% of the time), particularly in lymphohaematological disorders and, more recently, in AIDS (4, 5). The infection is believed to originate in subpleural foci and then to spread to ipsilateral hilar lymph nodes, much like tuberculosis. It heals spontaneously in most cases but sometimes, in immunocompromised and occasionally in immunocompetent patients, it spreads to other parts of the body $(5,6)$. It occasionally spreads to the central nervous system and causes meningitis, a condition commonly associated with pulmonary occult infections $(5,6)$. It is often not evident on a chest X-ray due to the spontaneous recovery of lung lesions (7). 


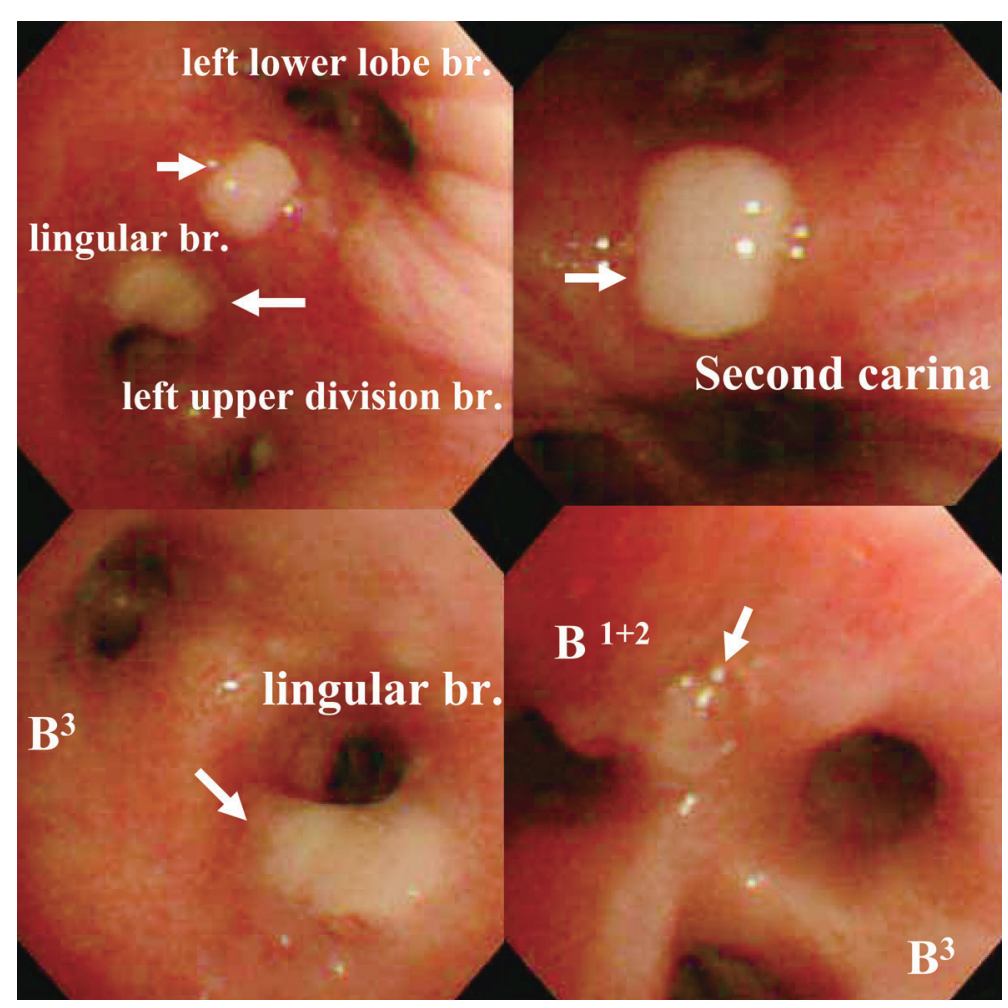

Figure 3. Bronchoscopic examination revealed 3 white elevated lesions (arrows) in the upper lobe bronchus of the left lung (at the spur of the upper lobe bronchus, at the orifice of the lingular and at the orifice of the left upper division bronchus $\left(B^{1+2}\right)$ ).

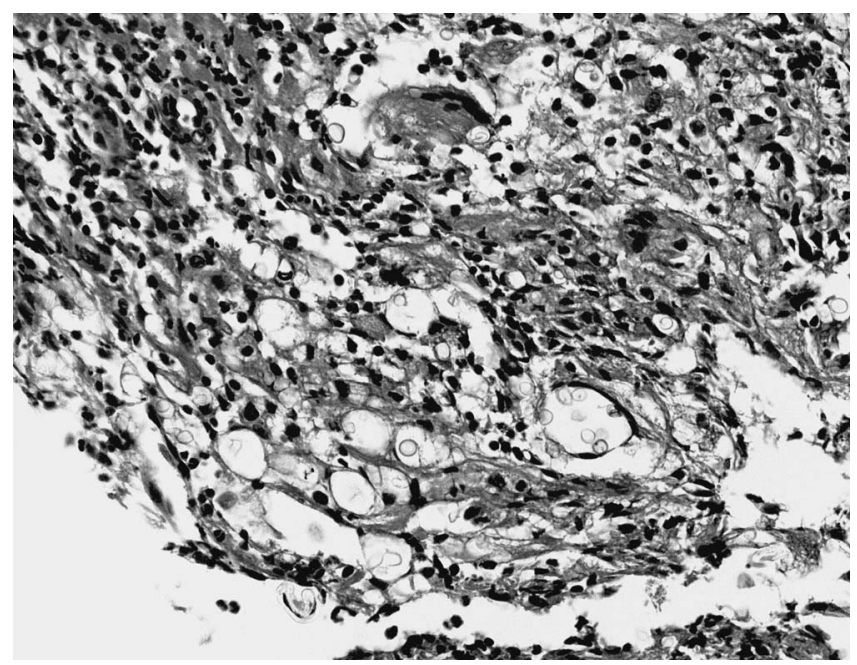

Figure 4. A histopathological examination of the transbronchial biopsy specimens revealed the presence of granulomatous lesions, including cryptococcal organisms, severe neutrophils infiltration, and some epithelioid cells and Langhans's giant cells (hematoxylin-eosin, original $\times 400$ ).

Cryptococcal lung lesions can differ, depending on the course of the underlying disease. A cryptococcal lung lesion is typically identified as a single, well-defined mass (often based in the pleura), as multiple nodules, or as a welldefined consolidation. The latter is more common in im- munocompromised patients $(8,9)$. Radiological findings often mimic primary or metastatic malignancies, since cryptococcal lung lesions may manifest themselves as one or more pulmonary mass lesions.

In immunocompromised patients, some unusual features, such as cavitations, pleural effusion, and lymphadenopathy, may be present. Calcification, however, is extremely rare (8).

Endobronchial cryptococcosis causing complete lung collapse has, to the best of our knowledge, only been reported once (1). That patient had a complete left lung collapse, and a bronchoscopic examination revealed a mass lesion totally occluding the lower lobe bronchus of the left lung and partially occluding the upper lobe bronchus of the left lung. Another patient with an upper lobe consolidation of the right lung and a minor volume loss has also been reported (2). Another report (3) described a patient with a middle and lower lobe collapse of the right lung. This report stated that the lung collapse was due to a white, lobulated lesion (revealed in a bronchoscopic examination) that had completely obstructed the orifice of the bronchus intermedius (3).

Four other patients who had an endobronchial cryptococcal infection that did not lead to lung collapse have also been reported $(7,10-12)$. The first patient from this group had a middle lobe consolidation of the right lung. A bronchoscopic examination of this patient revealed a haemorrhagic lesion covering the carina that caused a subtotal obstruction of the main bronchus of the right lung and a par- 


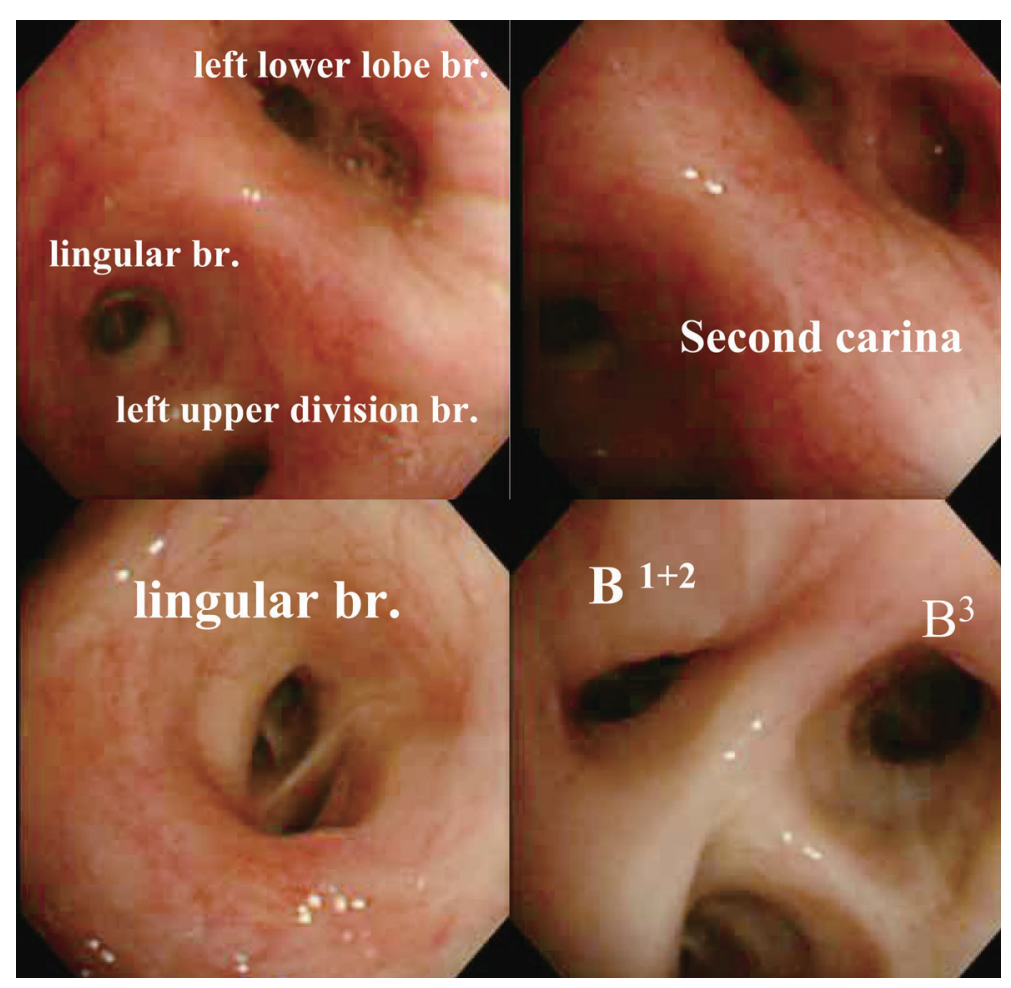

Figure 5. Bronchoscopic examination after 4 weeks of treatment revealed that the white elevated lesions in the upper lobe bronchus of the left lung had disappeared.

Table 3. Summarized Previous Case Reports of Endobronchial Cryptococcosis

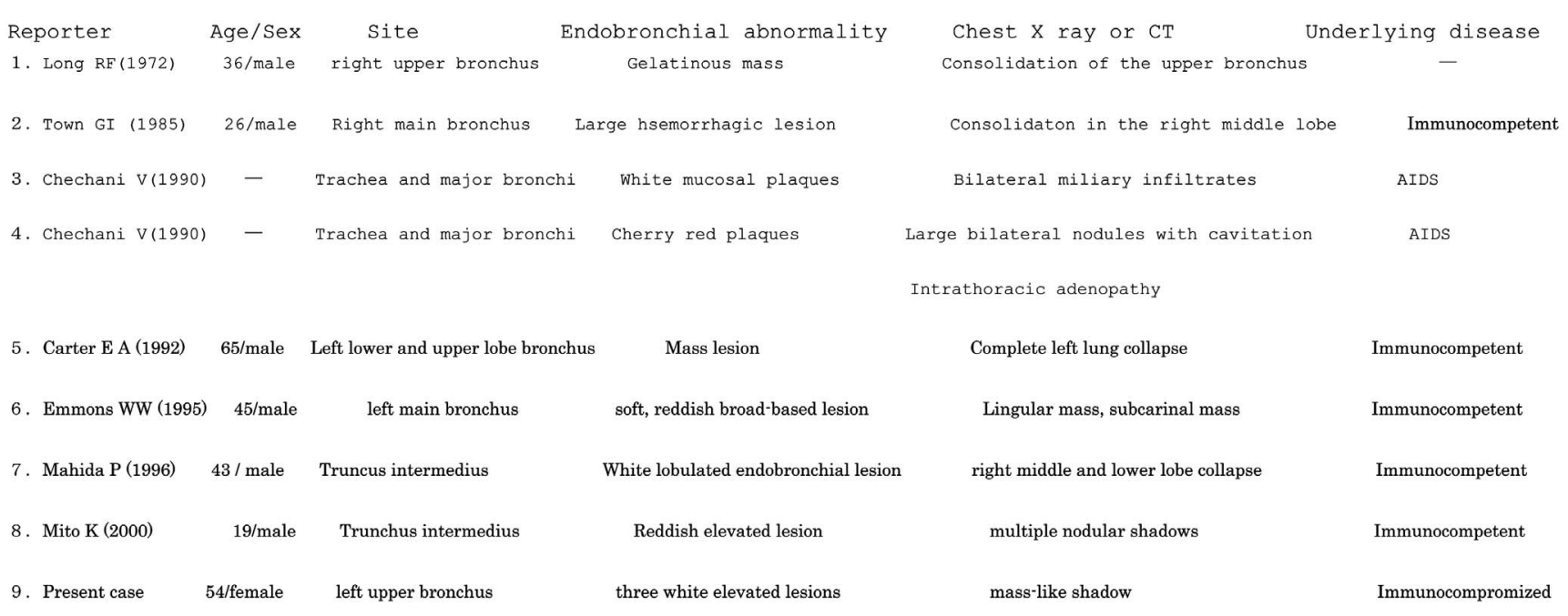

tial obstruction of the main bronchus of the left lung (7). The second patient from this group had a mediastinal mass and infiltration of the trachea and the main bronchi of the right and left lung (10). The third patient from this group had a lingular mass and was subjected to a segmental resection of the lingula. Following the resection, a chest CT scan revealed a mass in the subcarina. A soft, reddish, endobronchial lesion was observed on the medial wall of the main bronchus of the left lung (11). The fourth patient from this series had multiple nodular shadows in the right middle lung field that displayed a fusing tendency. Bronchoscopy revealed an elevated lesion in the truncus intermedius (12). The last 2 patients were reported to have a small and focal bronchial lesion, while the other 2 patients had large masses and displayed bronchial obstruction or compression. These 2 patients experienced direct bronchial infiltration from a lymph node, much like patients with endobronchial tuberculosis. We suspect that the infection in the present patient spread via a similar pathway.

Cryptococcosis presenting as an endobronchial lesion has 
rarely been described in 8 case reports (Table 3 ). White or reddish lesions were found as endobronchial abnormalities. The pathogenesis of endobronchial lesion may involve direct invasion from the intrathoracic lymph nodes like endobronchial tuberculsis (cases 4, 6, 8), and endobronchial cryptococcosis also can occur by direct extension from parenchymal involvement to cause endobronchial lesions (cases 1, 2, $5,7)$. In the present case, chest CT revealed left hilar and mediastinal lymphadenopathy. The route through the lymphatic system may therefore play an important part in the progression of cryptococcosis to endobronchial lesion.

There is insufficient evidence available to link cryptococcal endobronchial lesions with the status of a patient's immune system. According to previous reports, the status of the immune system in 3 such patients was normal, in an- other 3 patients it was unknown, and in 1 patient a skin allergy was verified. Five cases were immunocompetent ; thus, endobronchial lesion of cryptococcosis does not seem to be associated with immunodeficiency. The present patient was immunocompromised because she had been taking prednisolone for a long period of time. According to her medical history, many pigeons had taken refuge in her garden. Thus, it is likely that she inhaled a large quantity of crytococcal organisms while she was in this vicinity.

This case report highlights the fact that cryptococcal lung infections mimic the radiological and bronchoscopic features of lung cancer. This case report also indicates that cryptococcosis should be considered as an alternate diagnosis when endobronchial lesions are detected.

\section{References}

1. Carter EA, Henderson DW, McBride J, Sage MR. Case report: Complete lung collapse-An unusual manifestation of pulmonary cryptococcosis. Clin Radiol 46: 292-294, 1992.

2. Long RF, Berens SV, Shambhag GR. An unusual manifestation of pulmonary cryptococcosis. Br J Radiol 45: 757-759, 1972.

3. Mahida P, Morar R, Goolam Mahomed A, et al. Cryptococcosis: an unusual cause of endobronchial obstruction. Eur Respir J 9 (4): 837-839, 1996.

4. Chandler FW. Fungal infections. In: Pulmonary Pathology. Dail DH, Hammer SP, Eds. Springer-Verlag, New York, 1988: 189-257.

5. Wasser L, Talavera W. Pulmonary cryptococcosis in AIDS. Chest 92: 692-695, 1987.

6. Salyer WR, Salyer DC, Baker RD. Primary complex of cryptococcosis and pulmonary lymph nodes. J Infect Dis 130: 74-77, 1974.

7. Town GI, Seeman R. Pulmonary cryptococcosis: A report of two cases and review of the literature. N Z Med J 98: 894-895, 1985.

8. Fraser RS, Pare PD, Muller NL, et al. Diagnosis of the Diseases of the Chest. 4th ed. WB Saunders, Philadelphia, 1999: 904-909.

9. Khoury MB, Godwin JD, Ravin CE, Gallis HA, Halvorsen RA, Putman CE. Thoracic cryptococcosis: Immunologic competence and radiologic appearance. AJR Am J Roentgenol 142: 893-896, 1984.

10. Sinha P, Naik KG, Bhagwat GP. Mediastinal cryptococcoma. Thorax 33: 657-659, 1978.

11. Emmons WW 3rd, Luchsinger S, Miller L. Progressive pulmonary cryptococcosis in a patient who is immunocompetent. South Med J 88 (6): 657-660, 1995.

12. Mito K, Kawano H, Yamakami Y, et al. Primary pulmonary cryptococcosis with endobronchial lesion. Nihon Kokyuki Gakkai Zasshi 38 (4): 302-306, 2000 (in Japanese).

(C) 2007 The Japanese Society of Internal Medicine http://www.naika.or.jp/imindex.html 